\title{
Electron-Proton Pairing at High Temperatures, Solar Flares, and the FIP Effect
}

\author{
L. K. Pande ${ }^{1,2}$ \\ ${ }^{1}$ Theory Group, School of Environmental Sciences, Jawaharlal Nehru University, New Delhi, India \\ ${ }^{2}$ C1/1206, Vasant Kunj, New Delhi, India \\ Email: lalit_pande@hotmail.com
}

Received 27 November 2015; accepted 3 January 2016; published 8 January 2016

Copyright (C) 2016 by author and Scientific Research Publishing Inc.

This work is licensed under the Creative Commons Attribution International License (CC BY). http://creativecommons.org/licenses/by/4.0/

(c) (i) Open Access

\begin{abstract}
We analyze the line data from solar flares to present evidence for the emission spectrum of the recently discussed electron-proton pairs at high temperatures. We also point out that since the pairing phenomenon provides an additional source for these lines-the conventional source being the highly ionized high- $Z$ atoms already existing in the solar atmosphere, we have a plausible explanation of the FIP effect.
\end{abstract}

\section{Keywords}

Electron-Proton Pairing at High Temperatures, Emission Spectra from Solar Flares, FIP Effect

\section{Introduction}

Some time back, a finite-temperature Schrodinger equation was obtained to describe the pairing of an electron and a proton in a medium of such particles at finite temperature [1]. This work was a follow-up of an earlier paper where the Coulomb potential was temperature-generalized for the first time [2]. In these papers the approach followed was somewhat intuitive, leaving open the question of a derivation from first principles. Hence, beginning with the Bethe-Salpeter equation in vacuum, such a derivation was given in [3]. In an approximation scheme, which should work at low particle densities and high temperatures, this equation gives a temperaturedependent bound state spectrum for the electron-proton pair. In the temperature range around $10^{6} \mathrm{~K}$, the deepest of the states in the spectrum have binding energies in the $\mathrm{keV}$ range and can withstand the background thermal agitation of the medium. The transitions from the short-lived excited states to the deepest ones in this spectrum lead to spectral lines in the soft X-ray region. An application of the approach to the flaring regions of the Sun therefore leads to the prediction of such lines in the flare spectra. In the present note, we report on this matter: 
the calculated lines at a certain temperature from three Balmer-like series are essentially all seen in the flare data. We also point out that, since many of these lines are identifiable with the lines which in the conventional approach are presumed to originate from the low first ionization potential (FIP) elements, our approach seems to provide an explanation of the FIP effect which has been extensively discussed in the literature, e.g., in [4] and [5]. The details are given in the following.

\section{The Finite-Temperature Schrodinger Equation (FTSE) and Its Solutions}

The equation for the bound state at temperature $T$, or the FTSE, is given by

$$
\left(W-\frac{p^{2}}{2 \mu}\right) \psi(\boldsymbol{p})=\frac{\alpha}{4 \pi^{2}} Q(W, p) \int \frac{\mathrm{d} q}{(\boldsymbol{p}-\boldsymbol{q})^{2}} \psi(\boldsymbol{q}),
$$

where $W$ is the binding energy,

$$
\alpha=\frac{e^{2}}{4 \pi \hbar c} \simeq \frac{1}{137}, \quad \mu=\frac{m_{a} m_{b}}{m_{a} m_{b}}
$$

with $m_{a}$ and $m_{b}$ as the electron and the proton mass, respectively. The function $Q(W, p)$ is given by

$$
\begin{aligned}
Q(W, p) & =\tanh \left[\frac{\beta}{2}\left(\mu_{a} W-\varepsilon_{p}^{a}\right)\right]+\tanh \left[\frac{\beta}{2}\left(\mu_{b} W-\varepsilon_{p}^{b}\right)\right] \\
& =\frac{a-\tanh \left(\beta p^{2} / 4 m_{a}\right)}{1-a \tanh \left(\beta p^{2} / 4 m_{a}\right)}+\frac{b-\tanh \left(\beta p^{2} / 4 m_{b}\right)}{1-b \tanh \left(\beta p^{2} / 4 m_{b}\right)}
\end{aligned}
$$

where

$$
\begin{aligned}
& a=\tanh \left(\beta \mu_{a} W / 2\right), \quad b=\tanh \left(\beta \mu_{b} W / 2\right) \\
& \mu_{a}=m_{a} /\left(m_{a}+m_{b}\right), \quad \mu_{b}=m_{b} /\left(m_{a}+m_{b}\right), \\
& \varepsilon_{p}^{a}=p^{2} / 2 m_{a}, \quad \varepsilon_{p}^{b}=p^{2} / 2 m_{b} \quad \beta=1 / k T
\end{aligned}
$$

and $k$ is the Boltzmann constant. Using the approximation

$$
\tanh \left(\frac{\beta p^{2}}{4 m_{a, b}}\right) \simeq \frac{\beta p^{2}}{4 m_{a, b}}
$$

we can write

$$
Q(W, p) \simeq \frac{d_{1}-d_{2} p^{2}}{1-d_{3} p^{2}} \equiv \tilde{Q}(W, p)
$$

where

$$
d_{1}=a+b, \quad d_{2}=\frac{1+a b}{4 \mu k T}, \quad d_{3}=\frac{a / m_{a}+b / m_{b}}{4 k T} .
$$

Equation (7) can be solved in this approximation to give [1]

$$
\begin{gathered}
\psi(\boldsymbol{p})=g_{l}(\rho) Y_{l}^{m}(\theta, \eta) ; \quad \rho=|\boldsymbol{p}| / p_{0}, \quad p_{0}^{2}=-2 \mu W, \\
g_{l}(\rho)=\cos ^{4}(\varphi / 2) \sum_{k=l}^{\infty} a_{k} P_{k, l}^{(2)}(\cos \varphi) ; \quad \varphi=2 \arctan (\rho)
\end{gathered}
$$

where $l$ is the angular momentum quantum number, $m$ the azimuthal quantum number and $P_{k, l}^{(2)}(\cos \varphi)$ are given in terms of Gegenbauer polynomials $C_{k-l}^{l+1}(\cos \varphi)$ :

$$
P_{k, l}^{(2)}(\cos \varphi)=\frac{\sin ^{l}(\varphi)}{k+1} C_{k-l}^{l+1}(\cos \varphi) ;
$$


the coefficients $a_{k}$ in Equation (8) obey a difference equation given by

$$
b_{k+1}+b_{k} \frac{2(k+1)}{(k+l+1)} \frac{\left[(k+1) c_{1}-c_{2}\right]}{\left[(k+1) c_{3}-c_{4}\right]}+b_{k-1} \frac{(k-l)}{(k+l)}=0 ; \quad b_{k}=0 \text { for } k<l
$$

where we have put

$$
a_{k} \frac{(k+l+1)}{(k+1)^{3}}\left[(k+1) c_{3}-c_{4}\right]=b_{k}
$$

The parameters $c_{i}$ are given by

$$
\begin{aligned}
& c_{1}=\left(1-d_{3} p_{0}^{2}\right), \quad c_{2}=\left(\frac{\lambda}{2 p_{0}}\right)\left(1-d_{2} p_{0}^{2}\right) \\
& c_{3}=\left(1+d_{3} p_{0}^{2}\right), \quad c_{4}=\left(\frac{\lambda}{2 p_{0}}\right)\left(d_{1}+d_{2} p_{0}^{2}\right) ; \quad \lambda=-\alpha \mu
\end{aligned}
$$

Equation (10) is a second order difference equation and will in general have two solutions; the dominant and the dominated. The eigenvalues of the equation are those values of $p_{0}^{2}$ (or $W$ ) for which the dominant solution vanishes (for a review see [6] [7]), and they can be computed easily by the Hill determinant method; see [8]-[10] and [1]. The generic computer program for such computations is already given in [9], and can be adapted to the present situation without any difficulty. On substituting the numerical values for $m_{a}$ (electron mass), $m_{b}$ (proton mass) and $\alpha$ (the fine structure constant), we can use the program to solve for $W$ at any given value of $T$. What emerges is a discrete spectrum for $W$ : for each $l$, we get an increasing set of $W$ values which can be labeled by a serial number $\mathrm{n}$ (which plays the role of the principal quantum number in the case of the hydrogen atom). The results are illustrated in Table 1 . The typical dimensions of the Hill determinant for stability of levels up to the third decimal place (in eV) are $k=400$ for $n=1, l=0$; $k=16,000$ for $n=10, l=0, k=80,000$ for $n=20, l=0$; and so on.

It is now important to make sure that the solutions obtained are indeed consistent with the approximation given in Equation (5). This is done by verifying that the expectation values of $\left\langle\beta p^{2} / 4 m_{a}\right\rangle$ over the desired solution $\psi(\boldsymbol{p})$ corresponding to any $W_{n, l}$ are quite small, say $<0.01$. If not so, the solution must be discarded. It may be noted that since $m_{a}$ is much smaller than $m_{b}$, it is sufficient to check that $\left\langle\beta p^{2} / 4 m_{a}\right\rangle \equiv\left\langle x_{a}\right\rangle$ is quite small. To do this, we have to first calculate. $\left\langle x_{a}\right\rangle$. The details are given in Appendix A. We quote here only the result:

$$
\left\langle x_{a}\right\rangle=\frac{\mu_{b}\left|W_{n, l}\right|}{2 k T} \frac{S_{2}}{S_{1}}
$$

where

$$
\begin{aligned}
& S_{1}=\sum_{k=l}^{M}\left[\frac{\Gamma(k+l+2)}{(k-l) !(k+1)} \frac{a_{k} a_{k}}{(k+1)^{2}}+\frac{\Gamma(k+l+3)}{(k-l) !(k+1)} \frac{a_{k} a_{k+1}}{(k+1)(k+2)^{2}}\right] \\
& S_{2}=\sum_{k=l}^{M}\left[\frac{\Gamma(k+l+2)}{(k-l) !(k+1)} \frac{a_{k} a_{k}}{(k+1)^{2}}-\frac{\Gamma(k+l+3)}{(k-l) !(k+1)} \frac{a_{k} a_{k+1}}{(k+1)(k+2)^{2}}\right]
\end{aligned}
$$

Note that $M$ in the summations $S_{1}$ and $S_{2}$ denotes the highest $k$ value for which $a_{k} \neq 0 .\left|W_{n, l}\right|$ is the magnitude of the binding energy at serial number $n$ and angular momentum $l$, corresponding to which $\left\langle x_{a}\right\rangle$ is being calculated. Note further that $\left|W_{n, l}\right|$ is already calculated from Equation (10) by the Hill determinant method, which also determines the value of $M$. This method, however, does not require an explicit knowledge of the coefficients $a_{k}$. The latter are therefore yet to be determined. This can be done conveniently by using the backward Miller algorithm [11] [12] which is reviewed in Appendix A, where we also record the steps that need to be taken to calculate $a_{k}$ and whence $\left\langle x_{a}\right\rangle$ can be calculated. We then find that the smallness criterion is satisfied 
Table 1. Energy eigenvalues $\left|W_{n, l}\right|$ of Equation (10) at temperature $T=4.26 \times 10^{6} \mathrm{~K}$ for $1 \leq n \leq 30$ and $l=0,1$, and 2 obtained via the Hill determinant method. Note that energy level $(n=1, l=0)$ will not be physically realizable at this temperature (see the text for details). Typical dimensions of the Hill determinant for stability up to the third place of decimal are: $k=$ $400(n=1, l=0) ; k=16,000$ for $(n=10, l=0)$.

\begin{tabular}{|c|c|c|c|}
\hline \multirow{2}{*}{$n$} & \multicolumn{3}{|c|}{ Binding energy $\left|W_{n, l}\right|(\mathrm{eV})$} \\
\hline & $\left|W_{n, 0}\right|$ & $\left|W_{n, 1}\right|$ & $\left|W_{n, 2}\right|$ \\
\hline 1 & 5003.429 & & \\
\hline 2 & 929.218 & 934.085 & \\
\hline 3 & 360.299 & 360.398 & 360.421 \\
\hline 4 & 197.600 & 197.607 & 197.609 \\
\hline 5 & 125.586 & 125.587 & 125.587 \\
\hline 6 & 86.994 & 86.994 & 96.994 \\
\hline 7 & 63.845 & 63.845 & 63.845 \\
\hline 8 & 48.856 & 48.856 & 48.856 \\
\hline 9 & 38.591 & 38.591 & 38.591 \\
\hline 10 & 31.254 & 31.254 & 31.254 \\
\hline 11 & 25.827 & 25.827 & 25.827 \\
\hline 12 & 21.700 & 21.700 & 21.700 \\
\hline 13 & 18.489 & 18.489 & 18.489 \\
\hline 14 & 15.942 & 15.942 & 15.942 \\
\hline 15 & 13.887 & 13.887 & 13.887 \\
\hline 16 & 12.205 & 12.205 & 12.205 \\
\hline 17 & 10.811 & 10.811 & 10.811 \\
\hline 18 & 9.643 & 9.643 & 9.643 \\
\hline 19 & 8.655 & 8.655 & 8.655 \\
\hline 20 & 7.811 & 7.811 & 7.811 \\
\hline 21 & 7.085 & 7.085 & 7.085 \\
\hline 22 & 6.455 & 6.455 & 6.455 \\
\hline 23 & 5.906 & 5.906 & 5.906 \\
\hline 24 & 5.424 & 5.424 & 5.424 \\
\hline 25 & 4.999 & 4.999 & 4.999 \\
\hline 26 & 4.622 & 4.622 & 4.622 \\
\hline 27 & 4.285 & 4.285 & 4.285 \\
\hline 28 & 3.985 & 3.985 & 3.985 \\
\hline 29 & 3.715 & 3.715 & 3.715 \\
\hline 30 & 3.471 & 3.471 & 3.471 \\
\hline$\infty$ & 0.000 & 0.000 & 0.000 \\
\hline
\end{tabular}


for any given $W_{n, l}$ only if the temperature $T$ is above a certain value $T_{n}$, and furthermore, $T_{1}>T_{2}>T_{3}$...etc. The relevant results are displayed in Table 2 . We see from this table that the solution corresponding to $\mathrm{W}_{1,0}$ is acceptable only for $T>T_{1} \approx 10^{7} \mathrm{~K}$, that corresponding to $W_{20}$ or $W_{21}$ only for $T>T_{2} \approx 2.6 \times 10^{6} \mathrm{~K}$, and so on. Clearly, all the solutions corresponding to $W_{n, l}$ for $n>2$ are now acceptable if $T>T_{2}$. We must also keep in mind that a bound state with binding energy $W$ will survive in a medium at temperature $T$ only if $|W|$ is appreciably greater than (3kT/2), the latter being the average kinetic energy of thermal motion per particle in the medium. Thus, for instance, at a temperature $T=4.26 \times 10^{6} \mathrm{~K}$, the deepest possible states that can form in our spectrum are at the level $n=2$, i.e., $W_{2,0}$ and $W_{2,1}$. We note that for these levels $|W|>(3 k T / 2)$. So the states at these levels will not only form, but also survive in the hot medium. Of course the states with $n>2$ will increasingly have $|W|<(3 k T / 2)$ and will therefore behave as very short-lived excited states. The transitions from these $n>$ 2 excited states to the $n=2$ states should lead, in view of $\Delta l= \pm 1$ selection rule, to the following three Balmer- like series of spectral lines:

$$
\begin{gathered}
A:\left(W_{3,0}, W_{4,0}, W_{5,0}, \cdots\right) \rightarrow W_{2,1} \\
B:\left(W_{3,1}, W_{4,1}, W_{5,1}, \cdots\right) \rightarrow W_{2,0} \\
C:\left(W_{3,2}, W_{4,2}, W_{5,2}, \cdots\right) \rightarrow W_{2,1}
\end{gathered}
$$

The wavelength $\lambda$ of the spectral line corresponding to any transition involving the energy difference $|\Delta W|$ in $\mathrm{eV}$ is given by

$$
\lambda=\frac{h c}{|\Delta W|}=\frac{12398.4927}{|\Delta W|} \dot{\mathrm{A}} .
$$

Substituting for any allowed $|\Delta W|$ from the above series, and using numbers from Table 1, we can check that the resulting wavelengths fall in the soft $\mathrm{X}$-ray region.

\section{Application to Solar Flares}

Let us now apply our approach to Solar flares. The latter are appropriate for such an application as they occur in a medium with low particle densities $\left(\approx 10^{12} / \mathrm{cm}^{3}\right)$ at temperatures around a few million degrees Kelvin and are known to emit soft X-ray lines. Furthermore, since the flaring phenomenon is a prolonged affair, we may expect that between the initial (growing) phase and the final (decaying) phase, there should be a period over which the flare burns at a reasonably constant temperature (with fluctuations of, say, not more than $\pm 5000 \mathrm{~K}$ ). If we knew this temperature, we could calculate the spectral lines of the kind given by Equation (13) and then look for them in the soft X-ray line data from flares. Of course this temperature is not quite known to us. So what we could do is to calculate our spectral lines at different temperatures between $10^{6} \mathrm{~K}$ and $10^{7} \mathrm{~K}$, to see if there is any temperature in this range at which our calculated lines are reproduced in the flare data. As will be seen in the following, we find that there indeed is such a temperature given by $T=4.26 \times 10^{6} \mathrm{~K}$. The binding energy spectrum $\left|W_{n, l}\right|$ at this temperature is given in Table 1. From here, the wavelengths of spectral lines corresponding to series A, B, and C, given by Equation (13), can easily be calculated. They are given in Table 3 . The notation $\left(n^{\prime}, l^{\prime} \rightarrow n, l\right)$ means that the transition is from the level $W_{n^{\prime}, l^{\prime}}$ to $W_{n, l}$. These transitions are referred to in columns 2 and 6 and the corresponding calculated wavelengths are given in columns 3 and 7.

Table 2. Temperatures for decreasing values of $\left\langle x_{a}\right\rangle=\left\langle p^{2} / 4 m_{a} k T\right\rangle$ for several energy levels calculated via Miller's algorithm.

\begin{tabular}{cccccccccc}
\hline \multicolumn{7}{c}{$T\left(W_{n, 1}\right)$ : Temperature for energy level } & $\left|W_{n, 1}\right|\left(10^{6} \mathrm{~K}\right)$ \\
\hline$\left\langle x_{a}\right\rangle$ & $T\left(W_{1,0}\right)$ & $T\left(W_{2,1}\right)$ & $T\left(W_{2,0}\right)$ & $T\left(W_{3,1}\right)$ & $T\left(W_{3,0}\right)$ & $T\left(W_{4,1}\right)$ & $T\left(W_{4,0}\right)$ & $T\left(W_{5,1}\right)$ & $T\left(W_{5,0}\right)$ \\
\hline 0.20 & 4.0 & 1.05 & 0.875 & 0.436 & 0.38 & 0.229 & 0.209 & 0.141 & 0.126 \\
$\approx 0.10$ & 5.25 & 1.38 & 1.22 & 0.575 & 0.501 & 0.302 & 0.275 & 0.186 & 0.174 \\
$\approx 0.01$ & 10.9 & 2.63 & 2.63 & 1.20 & 1.20 & 0.650 & 0.650 & 0.398 & 0.398 \\
$\approx 0.0$ & 20 & 5 & 5.0 & 2.0 & 2.0 & 1.0 & 1.0 & 0.630 & 0.630 \\
\hline
\end{tabular}


Table 3. Calculated wavelength $\lambda(\dot{\mathrm{A}})$ at temperature $\mathrm{T}=4.26 \times 10^{6} \mathrm{~K}$ for the emission lines comprising the generalized Balmer series A, B, and C which characterize our pair spectrum. Except for a very small difference in wavelength for the first set of lines at $(3.0 \rightarrow 2.1)$ and $(3.2 \rightarrow 2.1)$, the series $A$ and $C$ are degenerate. Therefore for the rest of the transitions in these series, only those from the former are given in the table. The observed wavelengths (good to within $\pm 0.02 \dot{\mathrm{A}}$ ) are from the data on Solar flares by Phillips et al. [12]; those marked with (+) are from the data of McKenzie et al. [14].

\begin{tabular}{|c|c|c|c|c|c|c|c|c|}
\hline S. No. & $\begin{array}{l}\text { Transitions } \\
\text { (Series B) }\end{array}$ & $\begin{array}{c}\text { Calculated } \\
\lambda(\dot{\mathrm{A}})\end{array}$ & $\begin{array}{l}\text { Observed } \\
\lambda(\dot{\mathrm{A}}) \\
\text { to within } \\
\pm 0.02 \dot{\mathrm{A}}\end{array}$ & $\begin{array}{l}\text { Convention-al } \\
\text { source }\end{array}$ & $\begin{array}{c}\text { Transitions } \\
\text { (Series A \& C) }\end{array}$ & $\begin{array}{l}\text { Calculated } \\
\qquad \lambda(\dot{\mathrm{A}})\end{array}$ & $\begin{array}{l}\text { Observed } \\
\lambda(\dot{\mathrm{A}}) \\
\text { to within } \\
\pm 0.02 \dot{\mathrm{A}}\end{array}$ & $\begin{array}{c}\text { Conventional } \\
\text { source }\end{array}$ \\
\hline 1 & $3.1 \rightarrow 2.0$ & 21.797 & $21.798+$ & O VII & $\begin{array}{l}3.0 \rightarrow 2.1 \\
3.2 \rightarrow 2.1\end{array}$ & $\begin{array}{l}21.608 \\
21.613\end{array}$ & $\begin{array}{l}21.602+ \\
21.602+\end{array}$ & O VII \\
\hline 2 & $4.1 \rightarrow 2.0$ & 16.947 & $16.956+$ & $?$ & $4.0 \rightarrow 2.1$ & 16.835 & 16.821 & $?$ \\
\hline 3 & $5.1 \rightarrow 2.0$ & 15.428 & 15.428 & $?$ & $5.0 \rightarrow 2.1$ & 15.335 & - & - \\
\hline 4 & $6.1 \rightarrow 2.0$ & 14.721 & 14.737 & Fe XIX & $6.0 \rightarrow 2.1$ & 14.636 & - & - \\
\hline 5 & $7.1 \rightarrow 2.0$ & 14.327 & 14.311 & $?$ & $7.0 \rightarrow 2.1$ & 14.247 & 14.258 & Fe XVIII \\
\hline 6 & $8.1 \rightarrow 2.0$ & 14.083 & 14.076 & Ni XIX & $8.0 \rightarrow 2.1$ & 14.006 & 14.017 & $?$ \\
\hline 7 & $9.1 \rightarrow 2.0$ & 13.921 & 13.934 & $?$ & $9.0 \rightarrow 2.1$ & 13.845 & 13.842 & $?$ \\
\hline 8 & $10.1 \rightarrow 2.0$ & 13.807 & 13.796 & Fe XIX & $10.0 \rightarrow 2.1$ & 13.733 & 13.738 & Fe XIX \\
\hline 9 & $11.1 \rightarrow 2.0$ & 13.724 & 13.719 & Ne VIII & $11.0 \rightarrow 2.1$ & 13.651 & 13.645 & Ne VIII \\
\hline 10 & $12.1 \rightarrow 2.0$ & 13.662 & 13.669 & Fe XIX & $12.0 \rightarrow 2.1$ & 13.589 & - & - \\
\hline 11 & $13.1 \rightarrow 2.0$ & 13.614 & 13.630 & $?$ & $13.0 \rightarrow 2.1$ & 13.541 & 13.551 & Ne IX \\
\hline 12 & $14.1 \rightarrow 2.0$ & 13.576 & - & - & $14.0 \rightarrow 2.1$ & 13.504 & 13.504 & Fe XIX \\
\hline 13 & $15.1 \rightarrow 2.0$ & 13.545 & 13.551 & Ne IX & $15.0 \rightarrow 2.1$ & 13.474 & 13.463 & Fe XIX \\
\hline 14 & $16.1 \rightarrow 2.0$ & 13.520 & 13.520 & Fe XIX & $16.0 \rightarrow 2.1$ & 13.449 & 13.446 & Ne IX \\
\hline 15 & $17.1 \rightarrow 2.0$ & 13.500 & 13.504 & Fe XIX & $17.0 \rightarrow 2.1$ & 13.429 & 13.426 & Fe XIX (?) \\
\hline 16 & $18.1 \rightarrow 2.0$ & 13.483 & 13.463 & Fe XIX & $18.0 \rightarrow 2.1$ & 13.412 & 13.402 & Fe XIX (?) \\
\hline 17 & $19.1 \rightarrow 2.0$ & 13.468 & 13.463 & Fe XIX & $19.0 \rightarrow 2.1$ & 13.397 & 13.4-2 & Fe XIX (?) \\
\hline 18 & $20.1 \rightarrow 2.0$ & 13.456 & 13.446 & Ne IX & $20.0 \rightarrow 2.1$ & 13.385 & 13.375 & Fe XVIII \\
\hline 19 & $21.1 \rightarrow 2.0$ & 13.445 & 13.446 & Ne IX & $21.0 \rightarrow 2.1$ & 13.375 & 13.375 & Fe XVIII \\
\hline 20 & $22.1 \rightarrow 2.0$ & 13.436 & 13.446 & Ne IX & $22.0 \rightarrow 2.1$ & 13.366 & 13.354 & Fe XVIII \\
\hline 21 & $23.1 \rightarrow 2.0$ & 13.428 & 13.426 & Fe XIX (?) & $23.0 \rightarrow 2.1$ & 13.358 & 13.354 & Fe XVIII \\
\hline 22 & $24.1 \rightarrow 2.0$ & 13.421 & 13.426 & Fe XIX (?) & $24.0 \rightarrow 2.1$ & 13.351 & 13.354 & Fe XVIII \\
\hline 23 & $25.1 \rightarrow 2.0$ & 13.415 & 13.402 & Fe XIX (?) & $25.0 \rightarrow 2.1$ & 13.345 & 13.354 & Fe XVIII \\
\hline 24 & $26.1 \rightarrow 2.0$ & 13.410 & 13.402 & Fe XIX (?) & $26.0 \rightarrow 2.1$ & 13.339 & 13.322 & Fe XVIII \\
\hline 25 & $27.1 \rightarrow 2.0$ & 13.405 & 13.402 & Fe XIX (?) & $27.0 \rightarrow 2.1$ & 13.335 & 13.322 & Fe XVIII \\
\hline 26 & $28.1 \rightarrow 2.0$ & 13.400 & 13.402 & Fe XIX (?) & $28.0 \rightarrow 2.1$ & 13.330 & 13.322 & Fe XVIII \\
\hline 27 & $29.1 \rightarrow 2.0$ & 13.396 & 13.402 & Fe XIX (?) & $29.0 \rightarrow 2.1$ & 13.326 & 13.322 & Fe XVIII \\
\hline 28 & $30.1 \rightarrow 2.0$ & 13.393 & 13.402 & Fe XIX (?) & $30.0 \rightarrow 2.1$ & 13.323 & 13.322 & Fe XVIII \\
\hline- & - & - & - & - & - & - & - & - \\
\hline 29 & $\infty \rightarrow 2.0$ & 13.343 & 13.354 & Fe XVIII & $\infty \rightarrow 2.1$ & 13.273 & 13.279 & Fe XIX \\
\hline
\end{tabular}


We now turn to the relevant experimental information. The data between 5 and $20 \dot{A}$ were obtained by Phillips et al. [13] from a flare on August 25, 1982 and from another flare on November 5, 1980. The work of Acton et al. [14] covers the range between 11 and $94 \dot{A}$. These observations were made on an M-class flare on July 13, 1982. They covered a much larger range of wavelengths than covered by Phillips et al., but missed out bunches of lines, especially between 12.82 and $13.45 \dot{A}$. McKenzie et al. [15] also provide useful data between 8 and 22 $\dot{A}$. In the range common to all these three groups, the data of [13] are the most exhaustive. As to the accuracy of the wavelengths quoted by these authors, Acton et al. estimate it to be not better than $\pm 0.02 \dot{\mathrm{A}}$. The other authors do not give direct estimates of their own, but their discussions suggest that it would not be safe to violate the limits set by Acton et al. Keeping this in mind, we now compare the emission lines from these data with those calculated from our pair spectrum at $T=4.26 \times 10^{6} \mathrm{~K}$. Any line from the data which is within $\pm 0.02 \dot{\mathrm{A}}$ of a calculated line is identified with the latter. We may mention here that a change of up to $\pm 5000 \mathrm{~K}$ does not make any change in our calculated wavelengths up to the third decimal place.

The experimental results in Table 3 (columns 4 and 8) are taken from Phillips et al. [13], except in the range not covered by them, i.e., for $\lambda(3.1 \rightarrow 2.0), \lambda(3.0 \rightarrow 2.1)$ and $\lambda(3.2 \rightarrow 2.1)$, which are from McKenzie et al. (the corresponding numbers from Acton et al. are $21.80 \dot{\mathrm{A}}$ and $21.60 \dot{\mathrm{A}}$, not different from those of McKenzie et al. within the accuracy limit of $\pm 0.02 \dot{A})$. An exceptional situation exists in the case of $\lambda(4.1 \rightarrow 2.0)$ : the line close to its calculated value, $16.947 \dot{\mathrm{A}}$, is not seen by Phillips et al., though it is within their range. The experimental value $16.956 \dot{\mathrm{A}}$ is from McKenzie et al.

A look at Table 3 will show that except for the single line at $\lambda(14.1 \rightarrow 2.0)=13.576 \dot{A}$ [the observed wavelength closest to which is $\lambda=13.551 \dot{\mathrm{A}}$, so that $\Delta \lambda=0.025$ which is just outside the accuracy limit of $\pm 0.02 \dot{\mathrm{A}}$ ], series $\mathrm{B}$ is observed in its entirety. The single missing line could also account for the line at $\lambda(12.0 \rightarrow 2.1)=$ 13.589 $\dot{\mathrm{A}}$ in Series A. For the latter series, however, two additional lines $\lambda(5.0 \rightarrow 2.1)$ and $\lambda(6.0 \rightarrow 2.1)$ are not observed. Other than this, all the lines in this series are also observed. Series $C$ is degenerate with Series A except for the first line $\lambda(3.2 \rightarrow 2.1)=21.613 \dot{\mathrm{A}}$, the counterpart of the line $\lambda(3.0 \rightarrow 2.1)=21.608 \dot{\mathrm{A}}$. These two lines are nearly degenerate and correspond to the observed line at $21.602 \dot{\mathrm{A}}$ [13] or $21.60 \dot{\mathrm{A}}$ [12].

\section{Possible Connection with the FIP Effect}

It may be noted that 29 distinct lines from the Solar flare data have been used up in the above as evidence for our generalized Balmer series. The data of course contain hundreds of lines over a wide range in the X-ray region. Our object is not to suggest that they all originate from our pairing mechanism. In fact, following the pioneering works of Grotrian and Edlen, we take it for granted that these lines follow from the so-called forbidden transitions in highly ionized high- $\mathrm{Z}$ atoms, the presence of which in stellar plasmas is a natural consequence of their having been formed in the interior of stars. The sources of a great many of these lines have accordingly been identified and the effort in that direction continues. Nevertheless, we may mention that, of the 29 lines from the data which we matched with our calculated lines, eight remain unidentified (marked [?] in Table 3) and two have questionable identification (marked [?] after the possible identification).

The conventional approach is thus not all-encompassing. In fact, the lack of proper identification in this approach of as many as eight (possibly 10) lines out of 29 does suggest that these lines may have a different origin. But what evidence could there be for such an additional origin for the other 19 lines which have been identified in the conventional approach? Interestingly enough, some evidence is indeed there. As already noted, it comes from what is called the FIP effect [4] [5]. The effect is widely observed, but we shall confine ourselves here to the part which relates to the emission lines from the Solar flares. According to conventional wisdom underlying the classical stellar atmospheres theory, the relative abundances of various elements in a star are not expected to show any variation in its upper layers (unlike in the interior where thermonuclear-process gradients exist). The relative abundance pattern for elements from the Solar photosphere should thus not be different from that obtained from the Solar flares or from the Solar coronal active regions. It turns out that this is not so. Elements with low FIP appear to be relatively more abundant in regions with $T \geq 10^{6} \mathrm{~K}$ than in the photosphere $(T \approx 6000$ $\mathrm{K})$. How does one infer this? One compares the relative intensity pattern of emission lines for the same elements from a) the high temperature sources like the flares and the coronal active regions and b) the photosphere. One finds that the intensities of lines corresponding to the low FIP elements from the former are anomalously enhanced. This may be interpreted to mean that these low FIP elements are relatively more abundant in the high temperature sources, which is in disagreement with the classical stellar atmospheres theory. Let us now look at 
the situation from the point of view of this study. We observe that of the 19 lines predicted by us, which are also identifiable as lines from known elements, almost all can be traced to a single low FIP element Fe (see Table 3) in its ionized state given by Fe XIX. In the light of the FIP effect, these are thus the lines which show anomalous enhancement. Clearly, such enhancement can now be attributed to an additional mechanism for their origin viz. the pairing mechanism considered herein. Note that such enhancement will not be possible for any lines originating from the photosphere which exists at a temperature of about $6000 \mathrm{~K}$ at which the pairing does not take place. This opens up the possibility of leaving undisturbed the generally accepted (and empirically corroborated) picture of the uniformity of relative abundances of elements in the upper reaches of the Sun.

It may also be pointed out that the detailed theoretical calculations [16] of the intensity ratios of lines, specifically from Fe XIX, do not seem to match with the observed data from the flares. This mismatch between theory and observation may be another pointer to the existence of an additional mechanism for the origin of the above lines.

\section{Conclusions}

In the context of the present study, we note that the generally accepted and empirically corroborated picture of the uniformity of elements in the upper reaches of the Sun, e.g., the photosphere and the Solar flares and coronal regions, would imply that the relative intensities of spectral lines from various elements would not show any significant variation from one region to another. It turns out that this is not so. The reason is: while 19 of the 29 lines in the data analyzed here and attributed to our pairing mechanism at $T=4.26 \times 10^{6} \mathrm{~K}$ can also be identified with those from a single low FIP elements Fe XIX, their intensities in the flares region are found to be anomalously enhanced as compared with the intensities of lines from the photosphere. It then makes sense to conclude that this enhancement is due to the existence of another mechanism operative in the region of flares, but not in the photosphere. As has been argued above, our pairing mechanism takes place at temperatures exceeding about a million $\mathrm{K}$ and not at temperatures around $5000 \mathrm{~K}$ that characterize the photosphere. We further note that 8 (possibly 10) of the 29 lines in the data analyzed above are not identifiable (or have questionable identification) on the basis of transitions from Fe XIX or any other elements. This lends support to the view that they may well be due to the additional pairing mechanism presented here.

Finally, we should note that while a recent review [17] gives several references to the experimental data on solar flares observed since 1996, the problem of the anomalous enhancement of the abundance of elements dealt with here continues to be unaddressed. To deal with these data via the approach presented here is our next task.

\section{Acknowledgements}

The author is grateful to Dr. Bernhard Haisch for pointing out the relevance of the FIP effect to the present work. He is also grateful to Dr. J.J. Drake and Dr. Anand Bhatia for providing some valuable information. Finally, it is a matter of great pleasure to thank Dr. G.P. Malik without whose encouragement the present investigation would not have been completed.

\section{References}

[1] Malik, G.P., Pande, L.K. and Varma, V.S. (1991) Astrophysical Journal, 379, 788-795. http://dx.doi.org/10.1086/170554

[2] Malik, G.P., Pande, L.K. and Varma, V.S. (1989) Pramana-Journal of Physics, 32, L89-L94.

[3] Pande, L.K. (1991) Pramana-Journal of Physics, 37, 39-45.

[4] Haisch, B., Julia, L., Saba, R. and Meyer, J.-P. (1996) In: Bowyer, S. and Malina, R.F., Eds., Astrophysics in the Extreme Ultraviolet, Kluwer Academic Publishers, Netherland, 511-518.

[5] Laming, J.M., Drake, J.J. and Widing, K.G. (1995) Astrophysical Journal, 443, 416-422. http://dx.doi.org/10.1086/175534

[6] Hautot, A. (1986) Physical Review D, 33, 437-443. http://dx.doi.org/10.1103/PhysRevD.33.437

[7] Znojil, M. (1986) Physical Review D, 34, 1224-1228. http://dx.doi.org/10.1103/PhysRevD.34.1224

[8] Biswas, S.N., Datta, K., Saxena, R.P., Srivastava, P.K. and Varma, V.S. (1971) Physical Review D, 4, 3617-3620. http://dx.doi.org/10.1103/PhysRevD.4.3617 
[9] Biswas, S.N., Datta, K., Saxena, R.P., Srivastava, P.K. and Varma, V.S. (1973) Journal of Mathematical Physics, 14, 1190-1195. http://dx.doi.org/10.1063/1.1666462 http://scitation.aip.org/content/aip/journal/jmp/14/9/10.1063/1.1666462

[10] Killingback, J.F. (1983) Microcomputer Quantum Mechanics. Adam Hilger, Bristol.

[11] Gautschi, W. (1967) SIAM Review, 9, 24-82. http://dx.doi.org/10.1137/1009002

[12] Malik, G.P., Malik, U. and Varma, V.S. (1993) Astrophysics and Space Science, 199, 299-315. http://dx.doi.org/10.1007/BF00613204

[13] Phillips, K.J.H., Fawcett, B.C., Kent, B.J., Gabriel, A.H., Leibbacher, J.W., et al. (1982) Astrophysical Journal, 256, 774-787. http://dx.doi.org/10.1086/159950

[14] Acton, L.W., Bruner, M.E., Brown, W.A., Fawcett, B.C., Schweizer, W. and Speer, R.J. (1985) Astrophysical Journal, 291, 865-878. http://dx.doi.org/10.1086/163125

[15] McKenzie, D.L., Landecker, P.B., Broussard, R.M., Rugge, H.R., Young, R.M., Feldman, U., et al. (1980) Astrophysical Journal, 241, 409-416. http://dx.doi.org/10.1086/158354

[16] Bhatia, A.K., Fawcett, B.C., Lemen, J.R., Mason, H.E. and Phillips, K.J.N. (1989) Monthly Notices of Royal Astronomical Society, 240, 421-444. http://dx.doi.org/10.1093/mnras/240.2.421

[17] Laming, J.M. (2015) Living Rev. Solar Physics, 12, 2. http://dx.doi.org/10.1007/lrsp-2015-2 


\section{Appendix A}

We evaluate here the expectation value $\left\langle x_{a}\right\rangle$, given by

$$
\left\langle x_{a}\right\rangle=\left\langle\frac{\beta p^{2}}{4 m_{a}}\right\rangle=\frac{\beta}{4 m_{a}} \int \psi^{*}(p) p^{2} \psi(p) \mathrm{d} p / \int \psi^{*}(p) \psi(p) \mathrm{d} p
$$

where, in view of Equations (8) in the text,

$$
\psi(p)=\psi(p, \theta, \eta)=\cos ^{4}\left(\frac{\varphi}{2}\right) \sum_{k=l}^{\infty} a_{k} P_{k, l}^{(2)}(\cos \varphi) Y_{l}^{m}(\theta, \eta),
$$

and $P_{k, l}^{(2)}(\cos \varphi)$ is given by Equation (9).

Upon using

$$
\rho=\frac{|p|}{p_{0}}, \rho^{\prime}=\frac{\left|p^{\prime}\right|}{p_{0}}, p_{0}^{2}=-2 \mu W>0, \lambda=-\alpha \mu, \cos \varphi=x,
$$

we obtain, after going through some elementary algebra,

$$
\int \psi^{*}(p) \psi(p) \mathrm{d} p=\frac{p_{0}^{3}}{16} \int_{-1}^{1} \mathrm{~d} x\left(1-x^{2}\right)^{l+1 / 2}(1+x) \sum_{k=l}^{\infty} \sum_{k^{\prime}=l}^{\infty} \frac{a_{k} a_{k^{\prime}}}{(k+1)\left(k^{\prime}+1\right)} C_{k-l}^{l+1}(x) C_{k^{\prime}-l}^{l+1}(x) .
$$

With the help of the standard result

$$
\int_{-1}^{1} \mathrm{~d} x\left(1-x^{2}\right)^{v-1 / 2} C_{n}^{v}(x) C_{m}^{v}(x)=\frac{\pi 2^{1-2 v} \Gamma(2 v+1)}{n !(n+v)[\Gamma(v)]^{2}} \delta_{n, m}
$$

and the recurrence relation

$$
x C_{k^{\prime}-l}^{l+1}(x)=\frac{1}{2\left(k^{\prime}+1\right)}\left[\left(k^{\prime}-l+1\right) C_{k^{\prime}-l+1}^{l+1}(x)+\left(k^{\prime}+l+1\right) C_{k^{\prime}-l-1}^{l+1}(x)\right],
$$

we then get

$$
\int \psi^{*}(p) \psi(p) \mathrm{d} p=\frac{p_{0}^{3}}{16} \frac{\pi 2^{-1-2 l}}{[\Gamma(l+1)]^{2}} S_{1}
$$

where

$$
S_{1}=\sum_{k=l}^{M}\left[\frac{\Gamma(k+l+2)\left|a_{k}\right|^{2}}{(k-l) !(k+1)^{3}}+\frac{\Gamma(k+l+3) a_{k} a_{k+1}}{(k-l) !(k+1)^{2}(k+2)^{2}}\right] .
$$

Proceeding in a similar manner, we also obtain

$$
\begin{aligned}
\int \psi^{*}(p) p^{2} \psi(p) \mathrm{d} p & =\frac{p_{0}^{5}}{16} \int_{-1}^{1} \mathrm{~d} x\left(1-x^{2}\right)^{l+1 / 2}(1-x) \sum_{k=l}^{\infty} \sum_{k^{\prime}=l}^{\infty} \frac{a_{k} a_{k^{\prime}}}{(k+1)\left(k^{\prime}+1\right)} C_{k-l}^{l+1}(x) C_{k^{\prime}-l}^{l+1}(x) \\
& =\frac{p_{0}^{5}}{16} \frac{\pi 2^{-1-2 l}}{[\Gamma(l+1)]^{2}} S_{2},
\end{aligned}
$$

where

$$
S_{2}=\sum_{k=l}^{M}\left[\frac{\Gamma(k+l+2)\left|a_{k}\right|^{2}}{(k-l) !(k+1)^{3}}-\frac{\Gamma(k+l+3) a_{k} a_{k+1}}{(k-l) !(k+1)^{2}(k+2)^{2}}\right] .
$$

We thus obtain [12]

$$
\left\langle x_{a}\right\rangle=\frac{\beta p_{0}^{2}}{4 m_{a}} \frac{S_{2}}{S_{1}}=\frac{\beta \mu_{b} \mid W_{n, l}}{2} \frac{S_{2}}{S_{1}}
$$


Note that $M$ is the summations in $S_{1}$ and $S_{2}$ denotes the highest $k$ value for which $a_{k} \neq 0$, and $\left|W_{n, l}\right|$ is the magnitude of the binding energy corresponding to principal quantum number $\mathrm{n}$ and angular momentum $\mathrm{l}$ for which $\left\langle x_{a}\right\rangle$ is being calculated. Note further that $\left|W_{n, l}\right|$ is already calculated vide Equation (10) by the Hill determinant method, which also determines the value of $M$. The method does not require an explicit knowledge of coefficients $a_{k}$, which can be determined by using the backward Miller algorithm [12]. To this end, we define

$$
r_{k-1}=\frac{b_{k}}{b_{k-1}}
$$

whence Equation (10) can be written as

$$
r_{k} r_{k-1}+A_{k} r_{k-1}+B_{k}=0
$$

where

$$
A_{k}=\frac{2(k+1)\left[c_{1}(k+1)-c_{2}\right]}{(k+l+1)\left[c_{3}(k+1)-c_{4}\right]}, B_{k}=\frac{k-l}{k+l} .
$$

We thus have

$$
\begin{gathered}
r_{k-1}=\frac{-B_{k}}{\left(A_{k}+r_{k}\right)}, \\
b_{k}=r_{k-1} b_{k-1} ; \quad l+1 \leq k \leq M .
\end{gathered}
$$

These equations have to be supplemented by

$$
r_{M}=0, \quad b_{l-1}=0 .
$$

Equation (A14a) then determines $r_{M-1}, r_{M-2}, \cdots, r_{l+1}, r_{l}$, and given $b_{l}$ (which can be taken as unity because the ratio $S_{2} / S_{1}$ above will be independent of this choice), $b_{l+1}, b_{l+2}, \cdots, b_{M}$ are determined via Equation (A14b). Equation (11) then enables the coefficients $a_{l+1}, a_{l+2}, \cdots, a_{M}$ to be calculated.

Thus knowing $W_{n, l}$ corresponding to any value of $T$ as calculated from Equation (10), and the corresponding values of $l$ and $M$, we can calculate the coefficients $a_{k}$ and, finally, using Equation (A10), the value of $\left\langle x_{a}\right\rangle$. 